\title{
Manejo de emociones negativas en el aprendizaje colaborativo en linea. Enfoque multimodal para resolver dificultades técnicas
}

\author{
Simone Belli \\ Atracción de Talento Modalidad I \\ Departamento de Antropología Social y Psicología Social \\ Universidad Complutense de Madrid \\ Campus de Somosaguas, s/n \\ 28223 Pozuelo de Alarcón (Madrid) \\ E-mail: sbelli@ucm.es
}

MANEJO DE EMOCIONES NEGATIVAS EN EL APRENDIZAJE COLABORATIVO EN LÍNEA. ENFOQUE MULTIMODAL PARA RESOLVER DIFICULTADES TÉCNICAS

RESUMEN: El propósito de este artículo es identificar cómo los participantes manejan las dificultades técnicas durante el aprendizaje colaborativo en linea. Analizamos el marco de participación en un corpus compuesto por 30 horas de aprendizaje colaborativo en linea entre estudiantes de una universidad andina, su profesor y expertos internacionales. Presentamos una interacción multimodal del lenguaje verbal y corporal en una actividad de colaboración para el análisis momento a momento de la interacción social que evoluciona. También nos enfocamos en las formas en que los participantes interactúan con sus palabras y su expresión no verbal, utilizando el análisis de conversación. Hemos observado cómo las dificultades técnicas generan malestar social y emociones negativas entre participantes. Nosotros describimos cómo se muestran las emociones negativas en diferentes contextos y cómo los usuarios resolvieron esto durante el aprendizaje colaborativo en linea.

PALABRAS CLAVES: aprendizaje colaborativo en linea; problemas técnicos; interacción multimodal; emociones negativas; transformación digital.

SUMARIO: 1. Introducción. 2. Métodos. 3. Análisis: emociones negativas en contextos en línea. 4. Análisis: marco de participación. 5. Discusión.
NEGATIVE EMOTIONS MANAGEMENT IN COLLABORATIVE ONLINE LEARNING. MULTIMODAL APPROACH TO SOLVE TECHNICAL DIFFICULTIES

ABSTRACT: The purpose of this paper is to identify how participants manage technical difficulties during online collaborative learning. We analyze the participation framework in a corpus composed of 30 hours of online collaborative learning among students at an Andean university, their professor, and international experts. The internet-based IT platform used was ZOOM. We present a multimodal interaction of verbal and body language in collaborative activity for the analysis of moment-by-moment evolving social interaction. Also using conversation analysis, we focus on the ways in which participants interact with their words and their non-lexical expression. Thanks to this methodology, we describe the moment-by-moment interactional work performed in collaborative activity. We have observed how technical difficulties generate social unrest and negative emotions shared among participants. In many cases, these difficulties generate conflicts between participants. We describe how negative emotions are shown in mixed contexts, and how users solved these during online collaborative learning.

KEY WORDS: online collaborative learning; technical problems; multimodal interaction; negative emotions; digital transformation.

SUMMARY: 1 . Introduction. 2. Methods. 3. Analysis: negative emotions in online contexts. 4. Analysis: Participation framework. 5. Discussion.
GÉRER LES ÉMOTIONS NEGATIVES DANS L'APPRENTISSAGE COLLABORATIF EN LIGNE. APPROCHE MULTIMODALE POUR RÉSOUDRE LES DIFFICULTÉS TECHNIQUES

RÉSUMÉ : L'objectif de cet article est d'identifier comment les participants gèrent les difficultés techniques lors de l'apprentissage collaboratif en ligne. Nous analysons le cadre de participation à un corpus composé de 30 heures d'apprentissage collaboratif en ligne entre des étudiants d'une université andine, leur professeur et des experts internationaux. Nous présentons une interaction multimodale du langage verbal et corpore dans une activité collaborative pour l'analyse de l'évolution des interactions sociales à chaque instant. En utilisant également l'analyse de conversation, nous nous concentrons sur la façon dont les participants interagissent avec leurs mots et leurs expressions non lexicales. Nous avons observé comment les difficultés techniques génèrent des troubles sociaux et des émotions négatives partagées entre les participants. Nous décrivons comment les émotions négatives sont montrées dans des contextes mixtes et comment les utilisateurs les ont résolues lors de l'apprentissage collaboratif en ligne.

MOTS CLÉS : apprentissage collaboratif en ligne ; problèmes techniques ; interaction multimodale ; émotions négatives ; transformation numérique.

SOMMAIRE : 1. Introduction. 2. Méthodes. 3. Analyse : émotions négatives dans des contextes en ligne. 4. Analyse Cadre de participation. 5. Discussion. 


\section{Manejo de emociones negativas en el aprendizaje colaborativo en linea. Enfoque multimodal para resolver dificultades técnicas}

\section{SimONE BELLI}

\section{INTRODUCCIÓN}

Durante las reuniones de aprendizaje colaborativo en línea (Kaye, 1992; McConell, 1994; Macdonald, 2003; O'Conaill et al., 1993) la conexión a internet a menudo no es lo suficientemente rápida y los usuarios experimentan dificultades técnicas críticas. El propósito de este artículo es identificar cómo los participantes a estas reuniones manejan esta. Analizamos el marco de participación en un corpus compuesto por 30 horas de aprendizaje en línea entre estudiantes de una universidad andina, su profesor y expertos internacionales. A lo largo de estas reuniones, los estudiantes presentan sus proyectos sobre innovación y espíritu empresarial a expertos en este campo con sede en una universidad del Reino Unido. La plataforma de TI basada en internet utilizada fue ZOOM. El marco multimodal para el manejo de estas situaciones crea gestos y lenguajes ambientales y una estructura consecuente con el entorno compartido que se enfoca en la atención de los participantes: la plataforma digital.

El objetivo de este artículo es observar la relevancia de una interacción precisa entre la gestión de conflictos y la interacción multimodal, acordes con estudios anteriores que han sugerido la relevancia de los gestos y el lenguaje para mostrar la orientación hacia la acción en curso (Goodwin, 1979, 1980, 1981; Heath, 1984; Mondada, 2006; Rossano, 2012; Ruusuvuori, 2001; Tiitinen y Ruusuvuori, 2014). También queremos observar las emociones que se generan en estos espacios digitales.

Los estudiantes usan su segundo idioma, el inglés, para comunicarse con los profesores y los expertos en la reunión, pero cuando experimentan problemas, cambian a su primer idioma, el español, para administrar el proyecto entre ellos. Los datos se transcribieron de acuerdo con un sistema para capturar los detalles auditivos de la conversación, diseñado por Gail Jefferson (Sacks et al., 1974), y un sistema para registrar gestos ideado por Goodwin (1981), donde no se debe transcribir todo lo visible en el video, solo lo que es analíticamente relevante.

Este material se ha analizado a través del analisis de interacción multimodal (Goodwin, 1981; Stivers, Sidnell, 2005; Mondada, 2006; Becvar, Hollan, Hutchins, 2008) centrandonos en el lenguaje verbal y corporal en actividad colaborativa (Chovil y Bavelas, 1997). Utilizando este tipo de analisis (Perakyla, 2004; Rossano, 2012; Tiitinen y Ruusuvuori, 2014), nos centramos en las formas en que los participantes interactúan con sus palabras y su expresión no verbal (mirada, gestos, prosodia). Gracias a esta metodologia, describimos el momento a momento del trabajo de interacción realizado en la actividad de colaboración (Perakyla \& Ruusuvuori, 2006; Goodwin, 1981, 2007). 
La práctica de interacción multimodal ha sido ampliamente estudiada en encuentros institucionales, por ejemplo, en entrevistas de noticias (Heritage, 1985), conferencias de reuniones (Barnes, 2007) y en encuentros de consejeria y terapia (Perakyla, Antaki, Leudar, 2008; Hutchby, 2005; Tiitinen y Ruusuvuori, 2014; Weiste y Peräkylä, 2013).

En este estudio hemos observado cómo las dificultades técnicas y la lenta conexión de internet generan emociones compartidas entre los participantes. En algunos casos, estas dificultades generan conflictos entre participantes. Describimos cómo se muestran las emociones negativas en contextos en línea y cómo los usuarios las resolvieron durante las reuniones.

\section{MÉtodos}

Analizamos el estrés, preocupación y malestar en un corpus compuesto de 30 horas de grabaciones de reuniones en linea entre estudiantes de una universidad andina con sus docentes y tutores. En este aprendizaje colaborativo en línea los estudiantes presentan sus proyectos en innovación y emprendimiento a expertos en este campo de universidades del Reino Unido. La plataforma de internet basada en TI utilizada fue ZOOM ${ }^{1}$. Las reuniones se grabaron en la misma plataforma de TI compuesta por 32 estudiantes, dos profesores de la universidad de acogida y dos expertos en innovación de una universidad del Reino Unido.

La secuencia de pasos que nos interesa es: (a) las emociones negativas compartidas por los participantes, (b) los participantes identifican el problema (es decir, la conexión lenta), (c) los participantes intentan manejar la situación y finalmente (d) encontrar una solución para gestionar el problema. Este es nuestro "movimiento paso a paso para dar consejos" siguiendo una línea de acción similar a la descrita por Heritage y Sefi (1992: 377-389).

Por medio de las emociones negativas que los participantes expresan, reconocemos los problemas técnicos que ocurren en el aprendizaje colaborativo. En este rango de emociones negativas causadas por la conexión de internet lenta y dificultades técnicas, nos enfocamos en el estrés, preocupación y malestar. Estas emociones negativas son causa de conflictos afectivos en las reuniones; resolución de conflictos, planificación o negociación, donde la ambigüedad de la información y el requisito de una rápida aclaración y retroalimentación son críticos para el éxito de la interacción (Daft y Lengel, 1984; Whittaker, 1992). La falta de retroalimentación de los profesores es sin duda una fuente importante de emociones negativas como la frustración (Hara y King, 1999). Entendemos estas emociones como una forma de acceder a los patrones de significado que los usuarios aplican en su relación con la tecnología (Fortunati y Vincent, 2009). A través de la tecnologia, las emociones se expanden, se moldean, se estereotipan, se rein-

\footnotetext{
${ }^{1}$ https://zoom.us/.
} 
ventan y también se sacrifican, ya que deben someterse a los límites tecnológicos y los idiomas (Merino, 2017). En un estudio anterior, Dziubinska y Opoka (2007) observaron cómo los estudiantes a menudo se encuentran aislados, inseguros, ansiosos y frustrados en la plataforma digital.

Trabajaremos en las emociones desde una perspectiva relacional, centrando nuestra atención en la relación con los demás y el contexto (CantóMilà, 2016). Al igual que en Hochschild (1979), observaremos cómo se crean ciertas emociones en ciertos contextos sociales, las llamadas "reglas del sentimiento".

\section{ANÁliSis: EMOCIONES NEGATIVAS EN CONTEXTO EN LÍNEA}

En esta sección mostramos una reunión de 5 estudiantes que se conectaron desde la biblioteca de su universidad, el profesor de innovación y emprendimiento desde su oficina y 2 expertos que se conectaron desde sus casas en el Reino Unido. Profesores y expertos organizan este tipo de reuniones cada viernes a las 16 horas durante todo el semestre. Cada viernes dos grupos de estudiantes tienen media hora cada uno para presentar sus proyectos de innovación y emprendimiento y recibir retroalimentación de los expertos.

Durante la primera reunión a las 16 horas, un estudiante del segundo grupo (programado a las 16:30) intenta conectarse y aparece un nuevo usuario en la ventana de la plataforma de TI (Imagen 1).

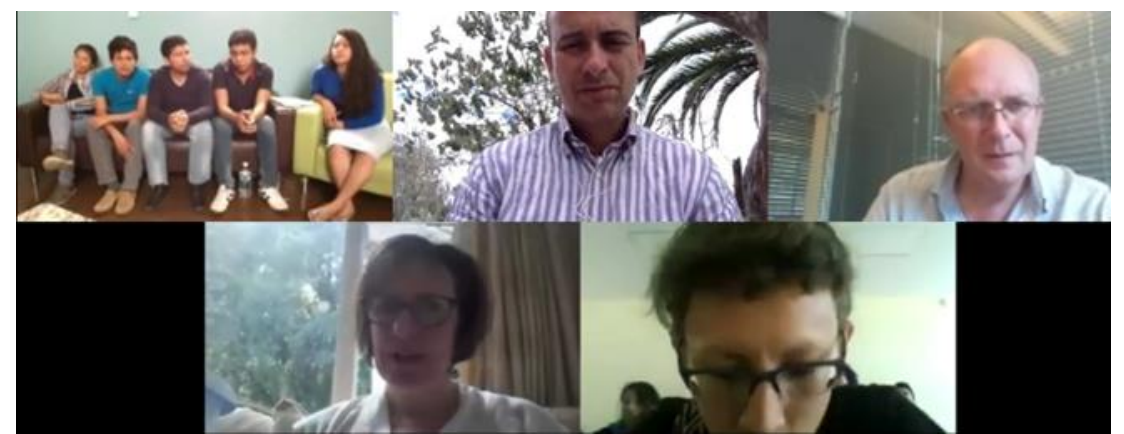

Imagen 1: Arriba a la izquierda: primer grupo de estudiantes; arriba en la mitad: profesor; arriba a la derecha: experto 1; abajo a la izquierda: experto 2; abajo a la derecha: estudiante del segundo grupo

En la ventana de abajo a la derecha un estudiante del segundo grupo de su clase trata de conectarse a la plataforma TI para saber si la infraestructura digital funciona correctamente. Mientras tanto, los participantes experimentan ciertos problemas con la conexión, la conversación no es fluida y los participantes empiezan a manifestar emociones negativas.

El profesor trata de comunicarse con el estudiante que no ha sido invitado a la sesión. El siguiente extracto fue sacado del chat de la plataforma de TI, por esta razón no está codificado con la transcripción de Jefferson. 


\section{Extracto 8:1}

(1) Profesor al estudiante del Segundo grupo: Hola

(2) vosotros no estás citado a esta reunión

(3) por favor, dejad el espacio libre

(4) estamos en una reunión

El profesor manifiesta su preocupación al no tener una reunión exitosa entre estudiantes y expertos debido a una conexión lenta. Finalmente, el estudiante no invitado entiende la situación y se desconecta de la plataforma de TI, pero los problemas en la reunión persisten.

Al mismo tiempo, mientras el profesor escribe al estudiante no invitado (extracto 8:1), el resto de los participantes experimentan emociones negativas y una conexión a internet lenta, lo que provoca un malestar social y compartido entre ellos.

\section{Extracto 7:9}

(5) ((Durante este extracto hay un eco muy fuerte, con un retraso de 0.7))

(6) Experto 1: Yeah (0.4) procesos (hhh) ambientales tienen: (1.0)

(7) Primer Grupo (Ángel): procesos (.) ambientales? (1.0)

(8) E1: ((Pone su mano derecha cerca de su oreja derecha y la mueve al frente.

(9) Imagen 2-3)) (0.2) > ruido chistoso <

(10) Profesor: Hay muchos ecos (0.8)

Durante esta primera parte de la reunión, un eco molestoso dificulta la conversación (extracto 7:9, línea 5). El experto 1 intenta dar retroalimentación a los estudiantes con casi ningún resultado (línea 6). En las líneas 7 y 8, observamos cómo el retraso en la comunicación provoca la repetición de las palabras. Los participantes están experimentando una situación de estrés causado por ecos. El estrés surge como un fenómeno logrado y una preocupación de los miembros, de cualquier manera, en que los miembros mismos se orientan hacia las nociones de "estrés". Al igual que en Merino (2017), los usuarios experimentan comodidad, frustración, nostalgia o estrés en su relación con la plataforma digital. Estas emociones negativas pueden afectar el aprendizaje y, en algunos casos, motivar el abandono del estudiante (Hara y King, 1999; Borges Sáiz, 2005).

Para expresar esta primera emoción negativa, E1 hace un movimiento con su mano para mostrar el escaso audio que experimenta desde su lugar (Imagen 2 - 3). El audio es un medio de comunicación rico y variado cuyo potencial va más allá de simplemente llevar palabras (Nardi et al., 1996), cuando esto es molesto o ruidoso, los participantes intentan encontrar una solución al problema utilizando partes de su cuerpo. 


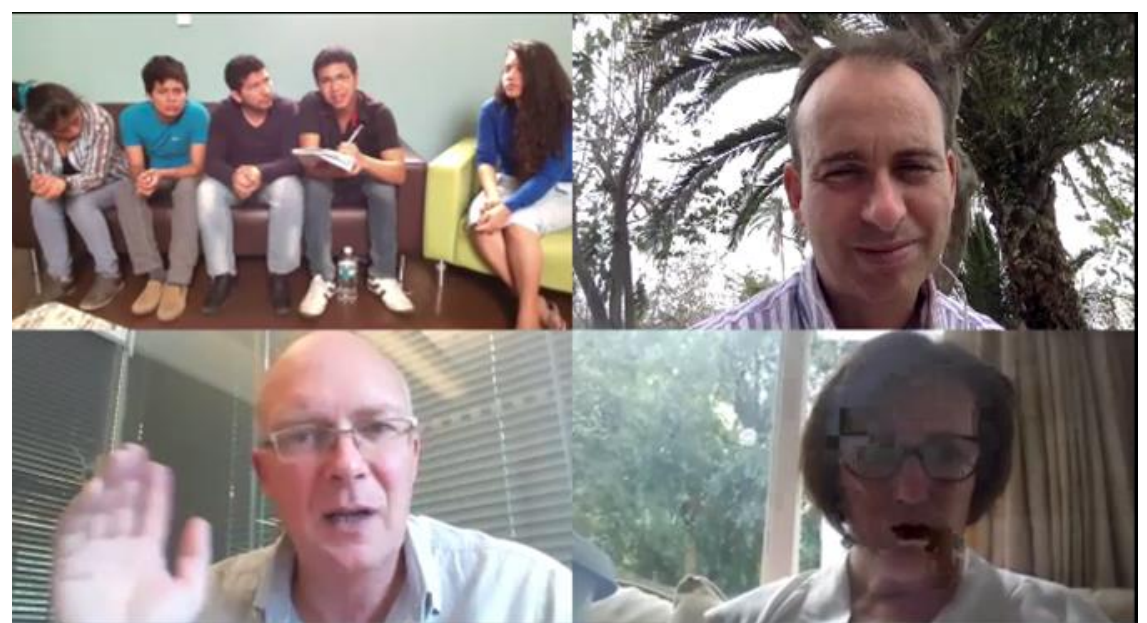

Imagen 2: Experto 1 pone su mano derecha cerca de la oreja derecha y la mueve hacia el frente (primera parte)

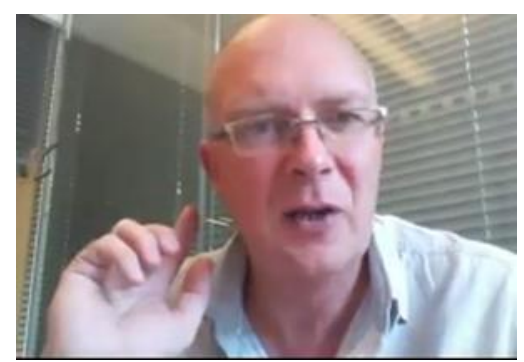

Imagen 3: Experto 1 pone su mano derecha cerca de la oreja derecha y la mueve hacia el frente (segunda parte)

En la línea 10 inmediatamente después el profesor repite las expresiones y el discurso del experto identificando el problema técnico que están experimentando: el eco. La organización secuencial de las interacciones dentro de la charla constituye lo que el profesor dice en la línea 10 como una descripción condicionalmente relevante del gesto del experto en la línea 8 (Schegloff, 1968; Sacks, 1992; Goodwin, 2007). El marco de participación (Goodwin, 2003b, 2007; Goodwin y Goodwin, 2004) constituido por el alineamiento mutuo de los cuerpos de los participantes crea un marco dinámico que fundamenta la conversación de manera indexada y encarna la acción que ocurre dentro de ella (Kendon, 1985).Para Goodwin (2007), la base del problema técnico es la base para el marco de atención conjunta, en el que múltiples actores asisten al mismo objeto en el entorno, lo que Tomasello (1999) ubica como central para la organización del lenguaje humano y la intersubjetividad.

La acción del profesor en las líneas 8 y 9 contiene un rango de formas de organización y regulación para la reunión (Goodwin, 2007). Gracias a la acción realizada, él puede manejar la conversación para resolver el problema mostrado por el experto al mover la mano. Los participantes comparten el 
estrés y el malestar. Ellos están llevando a cabo formas de acción conjunta para reunirse. Para seguir con la reunión es necesario manejar estas emociones negativas para continuar con la actividad colaborativa. El miedo a los escuchas y la grabación de videos no deseados puede reducir la efectividad de un sistema multimedia colaborativo, perjudicar la comunicación interpersonal y aumentar los niveles de estrés en el lugar de trabajo (Nardi et al., 1996). Para tener una reunión exitosa los participantes ponen atención a los detalles de la conversación (lineas 6-7). Siguiendo a Goodwin (2007), las palabras y los gestos están enmarcados por arreglos de los participantes que crean un enfoque compartido para la actividad.

Durante la reunión, los participantes continúan experimentando problemas técnicos, la conversación se interrumpe continuamente y los gestos mezclados con ecos se vuelven caóticos. La interacción conjunta entre los participantes cae, y la conversación es "desordenada". Los miembros de la reunión trabajan para establecer y mantener el orden, eso es lo que CA documenta exhaustivamente cuando la conversación parece "desordenada". Vemos métodos de conversación que se utilizan para tratar de manejar ese trastorno y repararlo de alguna manera. Tienden a ser construcciones del analista y no de las formas en que los propios miembros se orientan y explican el orden / desorden como asuntos de interacción entre ellos.

\section{Extracto 7:9 - 2}

(11) Estudiante A: ((Pone los dedos de su mano izquierda en la mitad de sus

(12) labios. Imagen 4)) (0.2) Es todo, bueno, es...

(13) E1: ¿Sí? OK

(14) A): >después después< la palabra:

(15) E1: Digo un ejemplo (.) un ejemplo de (.) de

(16) ((inaudible))

(17) Experto 2: ((se topa el cabello. Imagen 7)) Yo pienso ((inaudible)) Simon

(18) A): [((inaudible))] ((inaudible)) (1.2)

(19) Estudiante S: ((Se topa la barbilla por 40 segundos))

(20) E2: ((Pone su mano derecha en su cuello y permanece en esta posición

(21) Durante todo el extracto. Imagen 8))

(22) E1: ((Inaudible)) OK (0.7) el micrófono (1.5)

(23) ((Los estudiantes hablan español entre ellos)) (1.0)

Se identifica el problema (el micrófono, línea 22), pero se genera una situación crítica entre los participantes. Se vuelve muy dificil escucharse unos a otros, se pierde el diálogo principal, Las palabras inaudibles forman la mayor parte de la charla. Observamos cómo el profesor, que dirigió la reunión antes, ahora desaparece y no intenta entrar en el diálogo. 


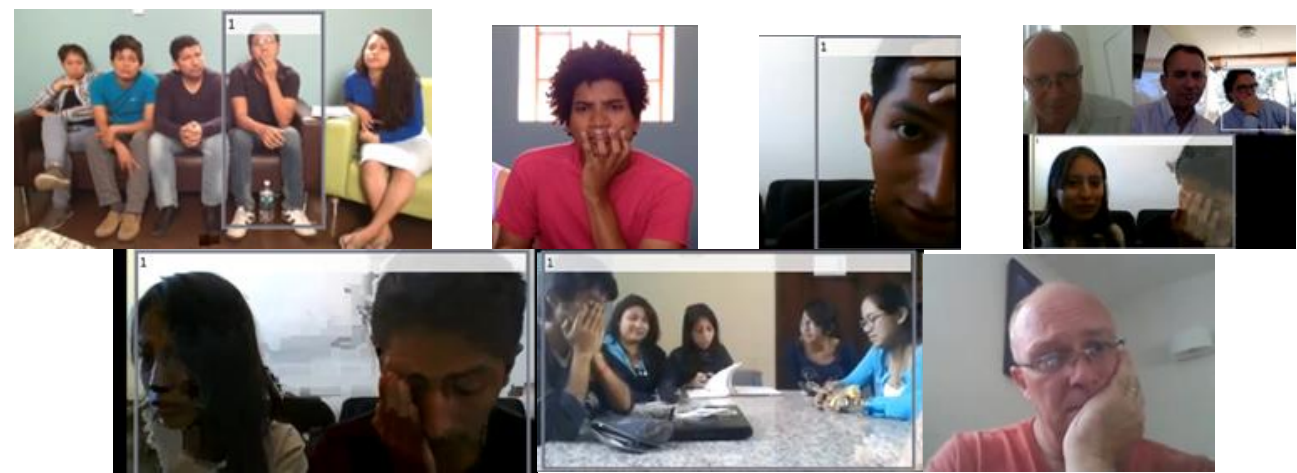

Imagen 4: Arriba a la izquierda, Ángel pone los dedos de su mano izquierda en la mitad de sus labios. Más ejemplos de gestos que expresan estrés durante la reunión

El gesto de tocar la cabeza con la mano se ha observado durante esta y otras reuniones (Imagen 4) donde la situación provocó estrés. Los gestos muestran el malestar social compartido por los usuarios y no ayudan a manejar la situación. La acción multimodal no es eficaz en esta parte de la reunión porque ocurre sin un marco de participación incorporado que crea una atención y acción visibles. Se modifican las configuraciones contextuales de un aprendizaje colaborativo en línea estándar.

Para Goodwin (2000a), una configuración contextual es un conjunto de diferentes tipos de fenómenos que los participantes consideran relevantes para la organización de la acción. Un aprendizaje colaborativo en línea (como una lectura colaborativa tradicional) necesita participantes, lenguaje y gestos, y un entorno compartido. Representan los detalles multimodales (Mondada, 2006) para analizar la interacción social entre los participantes (Imagen. 5). En esta parte de la reunión, los problemas técnicos son causados por el entorno compartido, una infraestructura técnica (computadora portátil, cámaras web, plataforma digital, conexión a Internet, etc.) que hace posible la actividad. Cuando algo de esta infraestructura no funciona correctamente, todo el entorno se cae y la actividad se cae. Los participantes intentan reparar este daño con palabras y gestos, los otros tres componentes de los detalles multimodales, para ofrecer una solución.

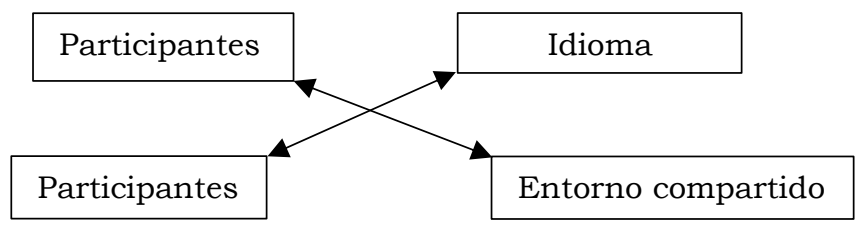

Imagen 5: Detalles multimodales para un aprendizaje colaborativo en línea

El grupo de estudiantes tiene una ventaja en el entorno compartido en comparación con el resto de los participantes: Ellos pueden hablar entre ellos en el mismo espacio físico y afuera de ese ambiente. Ellos comienzan a 
recibir retroalimentación a través de la modalidad cara a cara, administrando el problema técnico en una acción social fuera de línea. Cuando el experto 1 ofrece una posible causa del problema, comienzan a hablar entre ellos en su primer idioma, el español. El idioma, otro detalle multimodal, es otra herramienta adicional para que el grupo de estudiantes maneje el problema. Cambian de su segundo idioma a su primer idioma.

Esta organización interactiva de participación construida por la conversación y los gestos es la base de la actividad colaborativa. Representa una demostración del trabajo de los participantes y el ambiente para mostrar la cooperación en la realización conjunta de la actividad que se está realizando (Goodwin, 2007). Es necesario un enfoque de colaboración y cooperación entre los participantes para resolver el problema en esta reunión. En un aprendizaje colaborativo en línea, como en uno fuera de línea, la cooperación o la no cooperación en la participación solicitada para una actividad en particular proporciona un entorno compartido para la aparición visible de las emociones. Las emociones y habilidades para cooperar son más dificiles de mostrar cuando aparecen dificultades técnicas. El estrés se expresa con palabras y gestos para compartir estos sentimientos con el resto de los participantes.

\section{Extracto 7:9 - 3}

(24) (Llega un mensaje a Jordy y se acerca al ordenador para leerlo. (Imagen 6))

(25) Profesor: Hola Jordy

(26) Estudiante J: $\uparrow \mathrm{Si}(0.3)$

(27) P: necesitáis mejorar el sonido ((topa su frente con su mano derecha)).

(28) Imagen 6)) porque escuchamos mucho eco (0.3)

(29) J: Vale ((lee el mensaje del chat de E1)) Trata de silenciar tú, ¿OK?

(30) OK

(31) ((Amalia y Paul se topan las cabezas))

En este extracto aparecen dos conversaciones de chat, una del profesor y otra del experto 1. El entorno compartido, como vimos en el extracto 8: 1, es una plataforma multimodal para comunicarse entre los participantes, a través de la cámara web y el teclado. La cámara web y la reunión de video requieren una buena conexión a internet, pero la reunión en una sala de chat requiere una conexión normal. En una conversación escrita los usuarios usan teclados.

\section{Extracto 8:4}

(32) Profesor a estudiantes (privado): Hey! iiiNecesitáis mejorar vuestro sonido!!!

(33) Experto 1 (público): Trata de silenciar tu micrófono

(34) Estudiantes a profesor (en privado): AHORA????

(35) $\mathrm{P}$ a estudiantes (en privado): mucho mejor

En este extracto de chat, el profesor envía a los estudiantes en un chat privado el mismo mensaje que él ha compartido en la línea 27 del extracto 
7: 9 (mejora el sonido), cambiando el canal de comunicación. El Experto 1 adopta la misma estrategia, repitiendo la misma información en estos dos ambientes, chat y cámara web (el problema es el micrófono).

\section{ANÁLISIS: MARCO DE PARTICIPACIÓN}

En el aprendizaje colaborativo en línea los participantes adaptan sus estrategias en función de las dificultades técnicas. En nuestro caso, la cooperación comienza entre los participantes en una interacción multimodal. Los estudiantes se comunican utilizando cuatro canales diferentes:

- La reunión de video y voz entre cada participante (alto grado de dificultades de conexión);

- La sala de chat pública entre cada participante (bajo grado de dificultades de conexión);

- La sala de chat privada entre el profesor y ellos (bajo grado de dificultades de conexión);

- El espacio físico de la biblioteca (no afectado por dificultades de conexión).

La cooperación para gestionar el conflicto en una reunión es una interacción multimodal que utiliza diferentes plataformas y se mueve entre entornos en línea y fuera de línea. Esto demuestra la importancia de este marco para la organización de acción interactiva y del trabajo activo requerido para sostenerlo (Goodwin, 2007). Como dice Goodwin, la cooperación en la construcción de posturas corporales relevantes no es problemática en general. De hecho, los marcos de participación parecen diseñados específicamente para centrar la atención en los eventos que ocurren dentro de los marcos que crean, no en su propia organización (Kendon, 1985). Al igual que en Dziubinska y Opoka (2007), la comunicación efectiva entre estudiantes en una plataforma digital ayuda a crear un entorno de aprendizaje cooperativo. Prendergast (2003) afirma que las personas han fracasado cuando han tratado de producir cursos sin practicar en CSCL (aprendizaje colaborativo asistido por computadora).

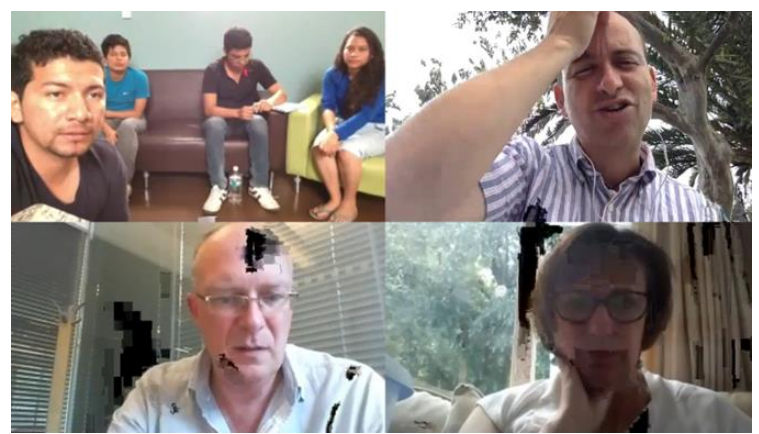

Imagen 6: Jordy se acerca a la computadora para leer los mensajes del chat. El profesor se topa la frente con su mano derecha 
En la imagen 6 Jordy está leyendo los mensajes de chat e intenta resolver el problema técnico. El profesor se toca la frente con la mano derecha, preocupado por resolver el problema y compartir información con Jordy a través de la conversación, el mensaje de chat y el gesto (Imagen 6). Jordy está respondiendo al profesor con "Sí" y "Vale", para confirmar que entiende cuál es el problema. "Vale" es una versión de "sí" como respuesta. Esta coparticipación en el marco contextual creado por la acción anterior de Jordy se muestra no solo en el contenido de lo que se dice en las lineas 29 y 30, sino también a través del comportamiento de los compañeros del grupo (Imagen 7).

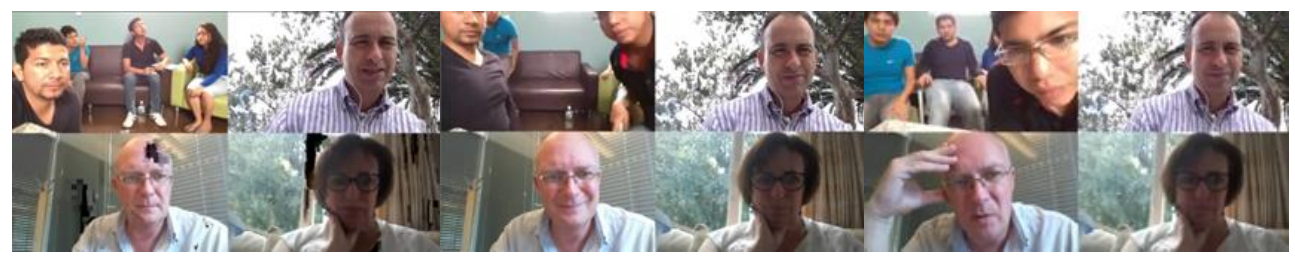

Imagen 7: Comportamiento de los compañeros del grupo

Mientras Jordy intenta resolver el problema técnico con el micrófono, Paul indica con su dedo izquierdo dónde se encuentra el módem en la sala de la biblioteca. Ángel sigue con la mirada la indicación. Más tarde, Ángel sigue a Jordy para acercarse a la computadora, la misma acción se repite con el resto de los compañeros de clase, Amalia y Paul. Rossano (2012) ha demostrado que la mirada está organizada en relación con la comprensión de los participantes sobre dónde se encuentran en una línea de acción. La dirección de la mirada de los participantes en línea, como fuera de línea, el aprendizaje colaborativo es relevante con respecto a la asociación entre formular y constituir la acción de tratar los problemas en el entorno compartido.

La mirada continua del estudiante hacia su compañero de clase muestra su orientación al curso de la acción que no se ha completado y su compromiso para recibir más información sobre el problema (Goodwin, 1981; Ruusuvuori, 2001; Rossano, 2012). El voltear la cabeza y las miradas desempeñan un papel fundamental en el cambio de orador (Duncan, 1972). Estos dos comportamientos dependen del enfoque (O'Conaill et al., 1993). Estas dos características especificas: enfocarse en los aspectos relevantes del problema de la expresión indicativa del problema precedente del usuario y la orientación a recibir más conversación relacionada al problema dejando un espacio luego de la formulación y la mirada a los participantes al momento de las transiciones, siguen la formulación y la confirmación de la respuesta de los compañeros de clase (Tiitinen and Ruusuvuori, 2014). Los oradores tienden a ser explícitos al momento de manejar el cambio de turno (O'Conaill et al., 1993). La alineación incorporada encontrada en la línea 29 del próximo extracto obedecerá a lo que el profesor y el experto 1 comunicaron entre ellos. 


\section{Extracto 7:9 - 4}

(36) Experto 1: OK >solo escuchen< (1.1) entonces ((inaudible))

(37) Estudiante J: Ahora no (4.0)

(38) (los compañeros de J se acercan a la computadora donde Jordy ha estado

(39) hasta ahora y comienzan a hablar entre ellos)

(40) (El eco desaparece y se resuelve el problema técnico) (1)

(41) Estudiante A: OK, OK señor, usted

(42) E1: (topa su frente con su mano derecha y permanece en esa posición

hasta el final del extracto. (Imagen 7)) Esto hago yo, el ejemplo de

(43) negocios ambientales donde:

La acción colaborativa es notable en la línea 39, los estudiantes han marcado una coparticipación en la actividad al aceptar que desean que el profesor y los expertos los ayuden a resolver el problema técnico. Los participantes deben saber cómo resolver problemas técnicos de manera colaborativa para no generar frustración o aislamiento en estas actividades de aprendizaje (Borges Sáiz, 2005).

En la línea 37 el experto 1 solicita a los estudiantes que solamente lo escuchen, pero Jordy no para su actividad de tratar de resolver el problema y continúa con la tarea. En la línea 38 él confirma que estaba continuando con su acción anterior y que no puede parar ahora. No es una batalla entre ellos o una falta de acción colaborativa, sino una ayuda para resolver el problema técnico, gracias a la colaboración de Ángel; es una actividad que se produce a través de la conversación y los gestos. Jordy y Ángel cooperan juntos para continuar una actividad colaborativa con el profesor y los expertos.

Al igual que en Serra (2015), la atención crítica y la participación emocional de los estudiantes los empoderan y mejoran las relaciones entre si, lo que el autor llama "respirar juntos". Los estudiantes se mueven más cerca de la computadora donde pueden comunicar adecuadamente lo que ambos, profesor y experto, proponen hacer entre ellos. Se mueven, se ponen de pie y administran la configuración de la computadora portátil (Imagen 7), algo que Goodwin (2007) clasifica como instrumental. En este caso, juntarse cerca de la computadora portátil es un éxito. La posición del cuerpo de la experta 2 en las imágenes 7-8-9 es completamente diferente. La experta 2 pone su mano cerca de la cabeza, manteniendo esta posición durante varios minutos, mostrando su preocupación debido a las dificultades técnicas.

Siguiendo el análisis de Goffman (1979) de la "postura" de cómo los participantes colocan sus cuerpos en una misma posición, podemos considerar cómo la Experta 2, después de algunas palabras, toca su cabello y luego pone una mano en su cuello (Imagen 8). Ella mantiene la misma posición hasta que se resuelva el problema. Para llevar a cabo líneas de acción relevantes, los participantes deben posicionarse para ver, sentir y percibir las actividades en curso (Goodwin, 2007). Ella arregla su postura precisamente para lograr esa atención de percepción relevante para compartir la preocupación en la reunión, el entorno y la percepción de los participantes. Estas 
"reglas de sentimiento" (Hochschild, 1979) la ayudan a sentirse a sí misma en relación con los demás, inmersa en el contexto relacional.

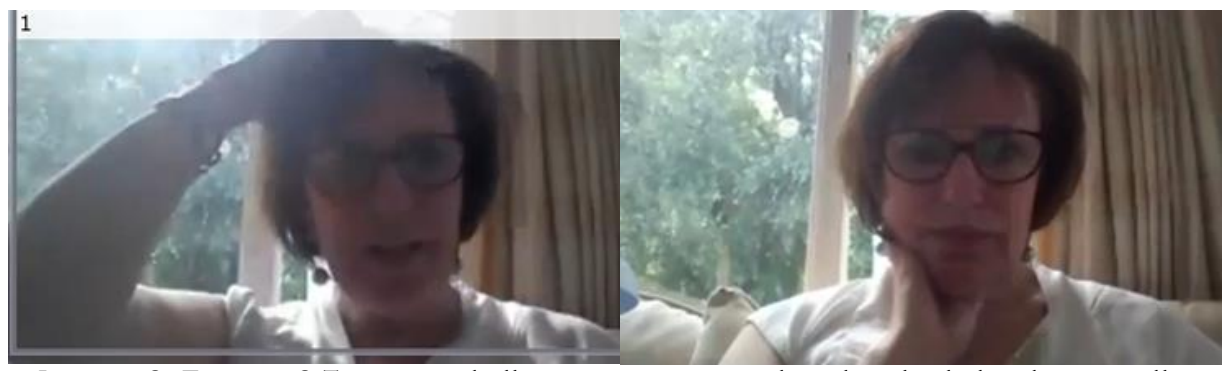

Imagen 8: Experta 2 Topa su cabello y pone su mano derecha alrededor de su cuello y mantiene esta posición durante todo el extracto

Goodwin (2007) sostiene que partes de la historia de la ciencia, la tecnología y la cognición distribuida han consistido en la construcción de herramientas que amplifican y sistematizan la percepción humana de un entorno que es el foco de la actividad de colaboración. Entonces, siguiendo esta idea, es posible describir cómo se siente la experta 2 sobre el entorno compartido digital y tecnológico y los problemas que causa a los participantes. Los participantes encarnan los problemas técnicos que sus cuerpos experimentan en el ambiente.

\section{Extracto 7:2}

(46) Profesor: Simon, $\uparrow$ Karen (0.2) preguntas:

(47) Experta 2: mmhh

(48) Estudiante A: (Coloca sus manos en la cabeza, rascándose)

(49) P: señalando (0.5)

(50) E2: (Empieza a rascarse la cabeza con su mano derecha, y lo hace

(51) por 9 segundos. (Imagen 9)) Sí tengo ciertos problemas $\uparrow$ entendiendo

(52) todo, mucho porque ehm la acústica en el cuarto (0.5) el

(53) equipo en... es... es un poco ehm pequeño, pequeño. (El estudiante que no fue

(54) invitado aparece en la ventana tratando de verificar si la plataforma

(55) funciona) dificil entender, pero, ehmm. Yo pienso eehhmm, yo pienso que tú

(56) estás >tratando de encontrar material<

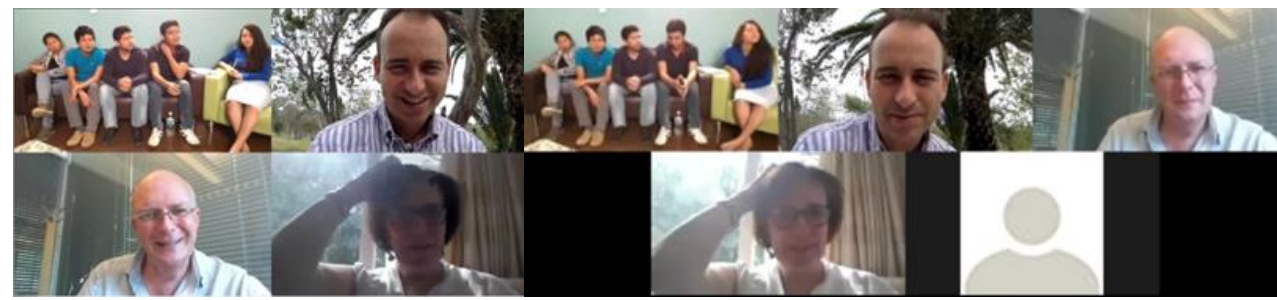

Imagen 9: Experta 2 se rasca la cabeza con su mano derecha por 9 segundos 
Este extracto aparece antes de los extractos que hemos presentado anteriormente $(7: 9$ - 4), pero nos es útil explicarlo de manera instrumental. Mientras se rascaba la cabeza, la experta 2 describe la imposibilidad de seguir la reunión por dificultades técnicas causadas por la conexión de los estudiantes. Puso la parte de su cuerpo (gesto) que aparece en el aprendizaje colaborativo en línea (entorno compartido) y explica lo que sucedió (lenguaje) para demostrar a los demás participantes que el posicionamiento incorporado es necesario para comprender lo que las dificultades técnicas provocan en ella, elaborando una adecuada alineación epistémica.

Situaciones como esta ocurren repetitivamente durante las reuniones, donde los participantes se alinean con la actividad de la manera que ella propone, y son relevantes para construir una postura de cooperación que hemos presentado en los extractos 7:9 - 3 y 7:9 - 4. Goodwin (2007) señala que alinearse adecuadamente con otros para construir los marcos de participación que organizan actividades de colaboración es absolutamente necesario para la cooperación que se está constituyendo.

El punto interesante en este marco de participación es la confianza que comparten todos los participantes. Para Garfinkel (1967) los esfuerzos por violar la confianza en las posturas cooperativas que son la base de la cognición y la acción mundanas se encontraron con una ira intensa, una de las emociones negativas que hemos observado durante nuestras reuniones y que ha acompañado a otras emociones negativas como el estrés. Goodwin (2007) encontró que la postura moral se hace visible cuando un actor se niega a asumir una postura de cooperación hacia las acciones iniciadas por otros y, por lo tanto, puede generar formas específicas de postura afectiva. Hemos observado cómo estas emociones negativas han causado esta situación en los extractos 8:1, 7:9 - 1 y 7:2. También hemos observado cómo los participantes han expresado emociones negativas que afectan la actividad de colaboración. El profesor y la experta 2 experimentan preocupación, el experto 1 experimenta malestar y los alumnos experimentan estrés. Todas las emociones negativas juntas produjeron un caos y una falta de cooperación en una primera etapa. Cuando los participantes examinan y entienden lo que está sucediendo (una dificultad técnica, conexión a internet lenta o problemas con el micrófono), intentan proporcionar un marco multipartito e interactivo que los incluya no solo como actores, sino también como receptores de la acción (ver tabla 1).

\begin{tabular}{|c|c|c|c|c|c|}
\hline Extracto & Linea & Actor & Destinatario & nosotros & $\begin{array}{c}\text { escuchamos } \\
\text { mucho eco }\end{array}$ \\
\hline $7: 9-3$ & 27 & tú & $\begin{array}{c}\text { Tienes que mejorar el sonido } \\
\text { porque }\end{array}$ & (yo) & entonces \\
\hline $7: 9-4$ & 37 & (tú) & Solo escucha & nosotros & $\begin{array}{c}\text { Estamos en } \\
\text { una reunión }\end{array}$ \\
\hline $8: 1$ & 2 & tú & $\begin{array}{c}\text { No estás citado a esta } \\
\text { reunión, por favor deja libre el } \\
\text { espacio }\end{array}$ & $\begin{array}{r}\text { Taba } \\
\hline\end{array}$
\end{tabular}

Tabla 1 
Los participantes utilizan la organización gramatical de las expresiones para quejarse de los estudiantes del primer grupo y del segundo grupo que no se ubican como individuos aislados, sino en la forma en que tratan a los demás dentro de la interacción. La interpretación del profesor de lo que los estudiantes del primer grupo y del segundo grupo están haciendo y mostrando con sus acciones actuales es consistente con el análisis anterior, que investiga la organización interactiva de los marcos de participación como un lugar primordial para la constitución de la acción humana (Goodwin, 2007). Tanto el profesor como los estudiantes están atrapados en una batalla donde cada uno insiste en el marco para la organización de la actividad. Pero las reuniones y discusiones se pueden resolver de múltiples maneras.

\section{Discusión}

Para Goodwin (2007), el hecho de no asumir tales posturas cooperativas puede llevar al estrés y las atribuciones que utilizan la forma en que un participante trata a los demás dentro de las interacciones como su punto de partida. Entonces entendemos cómo los marcos de participación en el aprendizaje colaborativo en línea son intrínsecamente multipartidistas. En la reunión los participantes encuentran útil ayudar a construir y sostener la actividad. Los marcos de participación sostienen la mutua orientación.

En el aprendizaje colaborativo en linea los participantes organizan su lenguaje y los gestos entre ellos de manera que establezcan un enfoque público y compartido de atención visual y emocional. Siguiendo a Goodwin (2007), podemos afirmar que la estructura visible de dichos marcos de participación permite a los individuos construir acciones conjuntas para que tomen en cuenta la estructura relevante en el entorno que es el enfoque de su trabajo y lo que hacen los demás. Como se ve en la imagen 7 , tales acuerdos son cruciales para la cooperación en una amplia variedad de escenarios.

Los estudiantes utilizan una formulación innovadora no para seguir el problema, sino por el contrario, para terminar el problema con los companeros de clase. Estudiantes, profesores y expertos utilizan conversaciones de chat para tratar de resolver un problema y continuar la reunión a través de video y audio.

La cooperación para gestionar el conflicto en un aprendizaje colaborativo en línea es una interacción multimodal. En este documento presentamos una dinámica en el aprendizaje colaborativo en línea con una lenta conexión a internet y dificultades técnicas para manejar los conflictos y las emociones negativas causadas por ellos. Se demostró que dos características de interacción son relevantes para esta dinámica: (a) Conflicto causado por problemas técnicos, y (b) conflicto manejado a través de la interacción entre usuarios. Hemos observado en este estudio cómo las dificultades técnicas generan emociones negativas entre los participantes. En muchos casos estas dificultades generan conflictos entre los participantes. 
Este estudio contribuye a este conocimiento previo sobre la importancia de la interacción multimodal en la visualización del compromiso y la organización de líneas de acción en la configuración de reuniones mediante el análisis de la construcción multimodal de una situación específica, es decir, un conflicto causado por problemas técnicos y administrado entre usuarios.

\section{REFERENCIAS}

BARNES, R. (2007): "Formulations and the facilitation of common agreement in meetings talk", Text \& Talk-An Interdisciplinary Journal of Language, Discourse Communication Studies, 27(3), pp. 273-296.

BECVAR, A.; HOLLAN, J. \& HUTCHINS, E. (2008): "Representational gestures as cognitive artifacts for developing theories in a scientific laboratory", $R e-$ sources, Co-Evolution and Artifacts, London: Springer, pp. 117-143.

BORGES SÁIZ, F. (2005): "La frustración del estudiante en línea. Causas y acciones preventivas", Digithum, 7, pp. 1-18.

CANTÓ-MILÀ, N. (2016): "Emotions from a relational perspective", Digithum, 18, pp. 23-42.

DAFT, R. \& LENGEL, R. (1984): "Information richness: A new approach to managerial behavior and organizational design", B. Straw \& L. Cummings (coord.), Research in organizational behavior, Greenwich, CT: JAI Press, pp. 191-223.

DUNCAN, S. (1972): "Some signals and rules for taking speaker turns in conversation", Journal of Personal and Social Psychology, 23, pp. 283-292.

DZIUBINSKA, M. \& OPOKA, J. (2007): "The online student as a driving force in the transformation of the Polish Virtual University", Digithum, 9, pp. 1-13.

FORTUNATI, L. \& VINCENT, J. (2009): "Introduction", Electronic emotion. The mediation of emotion via information and communication technologies. Bern: Peter Lang, pp. 1-31.

GARFINKEL, H. (1967): Studies in Ethnomethodology, New York: Prentice Hall.

GOFFMAN, E. (1979): "Footing", Semiotica, 25(1-2), pp. 1-30.

GOODWIN, C. (1979): "The interactive construction of a sentence in natural conversation", Everyday language: Studies in ethnomethodology, 97, pp. 101-121.

GOODWIN, M. H. (1980): "Processes of mutual monitoring implicated in the production of description sequences", Sociological inquiry, 50(3-4), pp. 303-317.

GOODWIN, C. (1981): "Conversational organization: Interaction between speakers and hearers", London: Academic Press.

GOODWIN, C. (2000): "Action and embodiment within situated human interaction", Journal of pragmatics, 32(10), pp. 14891522.

GOODWIN, C. (2003): "Pointing as situated practice", Pointing: Where language, culture and cognition meet, 41, pp. 217-241.

GOODWIN, C. (2007): "Participation, stance and affect in the organization of 
activities", Discourse \& Society, 18(1), pp. 53-73.

GOODWIN, C. \& GOODWIN, M. H. (2004). "Participation", A. Duranti (coord.) A Companion to Linguistic Anthropology, Oxford: Basil Blackwell, pp. 222-243.

HARA, N. \& KLING, R. (1999): "Students' frustration with a webbased distance education course", First Monday, 4, pp. 1231.

HEATH, C. (1997), "The analysis of activities in face to face interaction using video", Qualitative research: Theory, method and practice, 94, pp. 183-200.

HERITAGE, J. (2002), "The limits of questioning: Negative interrogatives and hostile question content", Journal of pragmatics, 34(10), pp. 1427-1446.

HERITAGE, J. \& SEFI, S. (1992): Talk at work, Cambridge: University Press.

HOCHSCHILD, A. (1979): "Emotion work, feeling rules and social structure", The American Journal of Sociology, 85, pp. 551575.

HUTCHBY, I. (2005): Media talk: Conversation analysis and the study of broadcasting. London: McGraw-Hill Education.

KAYE, A. R. (1992): Collaborative learning through computer conferencing. London: The Najaden Papers.

KENDON, A. (1985): "Some uses of gesture", en O. Tannen \& M. Saville-Troike (coord.), Perspectives on silence, Norwood, NJ: Ablex, pp. 215-234.

KENDON, A. (1995): "Gestures as illocutionary and discourse structure markers in Southern Italian conversation", Journal of pragmatics, 23(3), pp. 247-279.
MACDONALD, J. (2003): “Assessing online collaborative learning: process and product", Computers \& Education, 40(4), pp. 377391.

MCCONNELL, D. (1994): Implementing computer supported co-operative learning, London: Kogan Page.

MERINO, L. (2017): "Sobre el apego emocional a los teléfonos móviles en la vida cotidiana", Digithum, 19, pp. 37-46.

MONDADA, L. (2006): "Participants' online analysis and multimodal practices: projecting the end of the turn and the closing of the sequence", Discourse studies, 8(1), pp. 117-129.

NARDI, B., et al. (1996), "Video-asData: Technical and Social Aspects of a Collaborative Multimedia Application", Computer Supported Cooperative Work, 4, pp. 73-100.

O'CONAILL, D. et al. (1993), "Conversations over video conferences: An evaluation of the spoken aspects of video-mediated communication", Human-Computer Interaction, 8, pp. 398-428.

OCHS, E. (1979), "Transcription as theory", Developmental pragmatics, 10(1), pp. 43-72.

PERÄKYLÄ, A. (2004), "Two traditions of interaction research", British Journal of Social Psychology, 43(1), pp. 1-20.

PERÄKYLÄ, A. \& RUUSUVUORI, J. (2006), "Facial expression in an assessment", Video analysis methodology and methods, 2, pp. 1-19.

PERÄKYLÄ, A. ANTAKI, C. VEHVILÄINEN, S. \& LEUDAR, I. (2008): Conversation analysis and psychotherapy. Cambridge: Cambridge University Press.

ROSSANO, M. J. (2012): "The essential role of ritual in the transmission 
and reinforcement of social norms", Psychological Bulletin, 138(3), pp. 529-539.

RUUSUVUORI， J. (2001): “Looking means listening: coordinating displays of engagement in doctor-patient interaction", Social science \& medicine, 52(7), pp. 1093-1108.

SACKS, H.; SCHEGLOFF, E. A. \& JEFFERSON, G. (1974): "A simplest systematics for the organization of turn-taking for conversation", Language, 82, pp. 696-735.

SCHEGLOFF, E. A. (1968): "Sequencing in conversational openings", American anthropologist, 70(6), pp. 1075-1095.

SERRA, J. (2015): “A New Approach to Relationships in Live Music: Redefining Emotional Content and Meaning", Digithum, 17, pp. 1119.
STIVERS, T., \& SIDNELL, J. (2005): "Introduction: multimodal interaction", Semiotica, 156, pp. 120.

TIITINEN, S. \& RUUSUVUORI, J. (2014): "Using Formulations and Gaze to Encourage Parents to Talk About Their and Their Children's Health and Well-Being", Research on Language and Social Interaction, 47(1), pp. 4968.

TOMASELLO, M. (1999): "The human adaptation for culture", Annual review of anthropology, 32, pp. 509-529.

WEISTE, E. \& PERÄKYLÄ, A. (2013): "A comparative conversation analytic study of formulations in psychoanalysis and cognitive psychotherapy", Research on Language and Social Interaction, 46(4), pp. 299-321.

WHITTAKER, S. (1992): “Towards a theory of mediated communication", Unpublished manuscript. 